\title{
Spin Current Switching and Spin-Filtering Effects in Mn-Doped Boron Nitride Nanoribbons
}

\author{
G. A. Nemnes \\ "Materials and Devices for Electronics and Optoelectronics" Research Center, Faculty of Physics, University of Bucharest, \\ P.O. Box MG-11, Magurele-Ilfov 077125, Romania
}

Correspondence should be addressed to G. A. Nemnes, nemnes@solid.fizica.unibuc.ro

Received 2 August 2012; Accepted 3 September 2012

Academic Editor: Yong Guo

Copyright ( $\odot 2012$ G. A. Nemnes. This is an open access article distributed under the Creative Commons Attribution License, which permits unrestricted use, distribution, and reproduction in any medium, provided the original work is properly cited.

The spin transport properties are investigated by means of the first principle approach for boron nitride nanoribbons with one or two substitutional Mn impurities, connected to graphene electrodes. The spin current polarization is evaluated using the nonequilibrium Green's function formalism for each structure and bias. The structure with one Mn impurity reveals a transfer characteristics suitable for a spin current switch. In the case of two Mn impurities, the system behaves as an efficient spin-filter device, independent on the ferromagnetic or antiferromagnetic configurations of the magnetic impurities. The experimental availability of the building blocks as well as the magnitudes of the obtained spin current polarizations indicates a strong potential of the analyzed structures for future spintronic devices.

\section{Introduction}

The ongoing research in developing devices with highly efficient spin injection and manipulation turned the attention towards the low-dimensional diluted magnetic semiconductors (DMS). One promising candidate for a twodimensional DMS, recently investigated in several studies [1, $2]$, is represented by the Mn-doped boron nitride (BN) sheet. Hexagonal single-layer BN has been experimentally obtained using different techniques, such as micromechanical cleavage [3-6], layer-by-layer sputtering [7], ultrasonication [8], or by chemical [9] and thermal synthesis [10].

As indicated in [2], although bulk BN is a wide bandgap semiconductor, the Mn-doped BN sheets posses halfmetallic properties. This represents an important prerequisite for achieving good transport properties. Depending on the type of Mn substitution (i.e., on B or $\mathrm{N}$ atoms), different types of magnetic ordering are observed. However, the formation energies of the corresponding defects indicate that B-substitutions are more likely and the resulting ground state is ferromagnetic.
The $\mathrm{BN}$ sheet can be truncated in one direction to obtain a quasi-one-dimensional system called $\mathrm{BN}$ nanoribbon (BNNR). Depending on the direction of the truncation, there are two main types of BNNRs, namely, zigzag (zBNNR) and armchair (aBNNR). The BN sheet is an insulating isomorph of graphene-both hexagonal—with rather similar lattice constants, a fact which strongly supports integrating the two materials in an electronic device. Embedded atomic layers of BN in graphene were already successfully produced [11]. Hybrid BNC materials $[11,12]$ have also been investigated, revealing the importance of border states in the conduction properties as well as the spin-filter effects.

In this paper, the spin current switching and spinfiltering effects of $\mathrm{Mn}$-doped $\mathrm{BN}$ nanoribbons connected to metallic graphene electrodes are pointed out. Several samples are analyzed, starting with the undoped BNNR and systems with one and two magnetic impurities in the ferro- (FM) and antiferromagnetic (AFM) states. In the case of considered magnetic systems, the spin-resolved current indicates a high degree of polarization, which suggests potential applications as spin injectors. Besides the spin current switching and 
spin-filtering applications discussed here, the structure is important from the spin field-effect transistor (spinFET) perspective since the half metallicity of the BNNR segment ensures the possibility of a gate control over the transfer characteristics.

\section{Model and Computational Method}

The analyzed structures are depicted in Figure 1. The zigzag boron nitride nanoribbon (zBNNR) is connected to zigzag graphene nanoribbon (zGNR) electrodes. The zigzag structure was preferred to the armchair counterpart, since the zigzag graphene nanoribbon is always metallic, independent on its width. By contrast, armchair graphene nanoribbons (aGNRs) may present metallic or semiconducting behavior depending on the lateral size [13]. In this way, an efficient charge injection in the BNNR is ensured. The choice of graphene for the contact electrodes is also supported by the natural coupling between the two hexagonal lattices of zGNR and zBNNR. Both nanoribbons have hydrogen-passivated edges.

The current approach is based on first principle calculations using the density functional theory (DFT) as implemented in SIESTA [14], which has the advantage of linear scaling of the computational time with the number of atoms involved. This is achieved by using finite-support (localized) basis sets. For the exchange-correlation functional, the parametrization proposed by Ceperley and Alder is employed within the local spin density approximation (LSDA). The structural relaxations for both graphene and BN nanoribbons provide the lattice constants $a_{C}=1.42 \AA$ and $a_{\mathrm{BN}}=1.44 \AA$, respectively, which are consistent with the established experimental values. The distance from the hydrogen atoms to edge atoms in the nanoribbon is $d_{H}=$ 1.1 $\AA$. For these calculations, a Monkhorst-Pack scheme with $1 \times 1 \times 10$ mesh points in $\mathrm{k}$-space was used to perform integrations over the Brillouin zone. The structural relaxations were performed in the supercell approach implemented in SIESTA, until the interatomic forces were less than $0.04 \mathrm{eV}^{-1}$. The nanoribbons from adjacent supercells have enough separation for them not to interact. As depicted in Figure 1, the semi-infinite zGNR electrodes are constructed from the repetitive unit containing $16 \mathrm{C}$ atoms and $4 \mathrm{H}$ atoms, which account for the passivation. Inbetween the electrodes the zBNNR segment has one repetitive unit. The analyzed magnetic structures assume one or two Mn substitutions on boron atoms. Using constrained spin polarized DFT, the magnetic moments of the Mn impurities are set and for the two impurity cases it is possible to analyze the spin-resolved current in both ferro- and antiferromagnetic configurations.

Once the structural configurations are set, the spinresolved current is calculated in the framework of nonequilibrium Green's function formalism using the additional package TranSIESTA [15]. The system is partitioned into the left/right $(L / R)$ contacts and scattering region (SR). The Landauer formula gives the current calculated from the bias-dependent total transmission $T(E ; V)$ for each spin

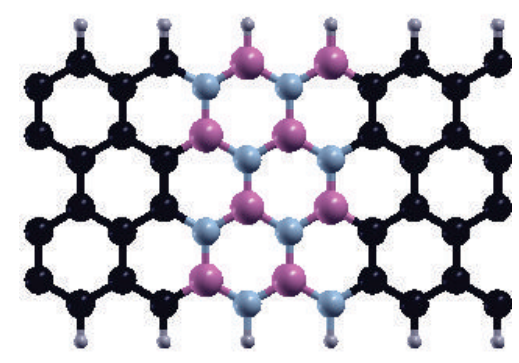

(a)

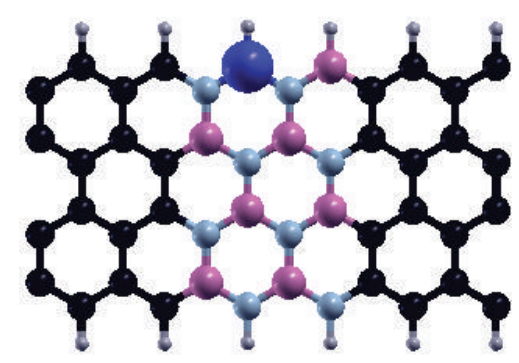

(b)

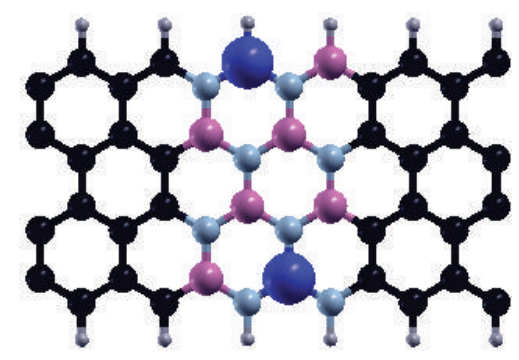

(c)

FIGURE 1: Simulated structures consisting of graphene electrodes (black) and the undoped (a), Mn-doped (b, c) zigzag boron nitride nanoribbon (pink, light blue). The Mn magnetic impurities (dark blue) substitute boron atoms. The nanoribbons are passivated with hydrogen (gray).

component (up/down), which, in the low temperature limit, becomes

$$
I(V)=\frac{2 e}{h} \int_{E_{F}-V / 2}^{E_{F}+V / 2} d E T(E ; V) .
$$

In the following, it is assumed that $E_{F}=0$ at zero bias, while at finite bias the two electrochemical potentials vary as $\mu_{L}=+V / 2$ and $\mu_{R}=-V / 2$. The transmission coefficient as a function of energy for each structure and each bias is obtained from

$$
T(E)=\operatorname{Tr}\left[\Gamma_{L}(E) G^{a}(E) \Gamma_{R}(E) G^{r}(E)\right],
$$

where $G^{a / r}$ are the advanced/retarded Green's functions and $\Gamma_{L / R}$ are self-energies corresponding to the two contacts.

The efficiency of a spin-filter device is characterized by the polarization of the spin current, defined as

$$
p=\frac{I_{\uparrow}-I_{\downarrow}}{I_{\uparrow}+I_{\downarrow}} .
$$




\section{Results and Discussion}

The first step in establishing the spin-filter capabilities of the considered structures is to analyze the spatial spin densities at zero bias. Making use of constrained spin DFT calculations, a maximum magnetic moment is applied for each $\mathrm{Mn}$ impurity. In the sample with two Mn impurities both ferroand antiferromagnetic spin configurations are considered. Figure 2 shows the localization of the spin density around the magnetic impurities and the polarization of neighboring atoms which are otherwise nonmagnetic. In the one magnetic impurity case, one can observe a polarization of the same type for the atoms located near the upper edge (see Figure 2(a)). The atoms at the lower edge, especially those in the conducting graphene electrodes are polarized oppositely, while the midsections including most of the zBNNR remain rather unpolarized. The picture changes when one additional impurity is added. In the two impurity case, it is clear that each $\mathrm{Mn}$ atom fixes the polarization of the atoms on the corresponding edge. From these two samples analyzed, one can already hint that the two impurities present in zBNNR should induce more pronounced spin-filter effects.

In order to account for the spin-filter efficiency, defined by (3), one has to calculate the transmission function $T(E)$ for considered samples at each bias point. In Figure 3, $T(E)$ is depicted for each spin component in the case of pristine zBNNR and for the other two magnetic samples already indicated. The transmissions are calculated for zero bias and for $U=1 \mathrm{~V}$, which is the maximum bias considered. For the pristine zBNNR, one obtains as expected equal transmission coefficients for both spin components and consequently there is no net polarization of the spin current. The small maximum at the Fermi energy is reminiscent of the transmission in a perfect graphene nanoribbon at zero bias. The situation changes with the introduction of magnetic impurities, as one can see in the (b,c,d) subplots. The rather sharp variations in the transmission functions are due to the intrinsic scattering between the propagating modes in the graphene electrodes, coupled to the BN nanoribbon with $\mathrm{Mn}$ impurities.

Calculations of the transmission functions are performed for each bias point starting with $0 \mathrm{~V}$ up to $1 \mathrm{~V}$ in steps of $0.1 \mathrm{~V}$. The polarization of the spin current extracted according to (3) is depicted in Figure 4. As already pointed out in the beginning of this section, the sample with two magnetic impurities is expected to produce a larger spin current polarization. Indeed, for only one impurity at biases below $0.3 \mathrm{~V}$ a negligible fraction of polarized current is observed. This is consistent with the image provided in in Figure 2(a), where a large section of the zBNNR remains unaffected by the presence of the Mn impurity. However, as the bias is increased above $0.3 \mathrm{~V}$, the potential drop along the zBNNR favors the action of the Mn impurity and the spin polarization current is significantly enhanced. Such a behavior is very important from the perspective of a spin current switch as a spintronic device, since it provides two regimes defined by low (off-state) and high (on-state) polarizations. Note that in this case the charge current is not subject to such an off-on transition and this is reflected in

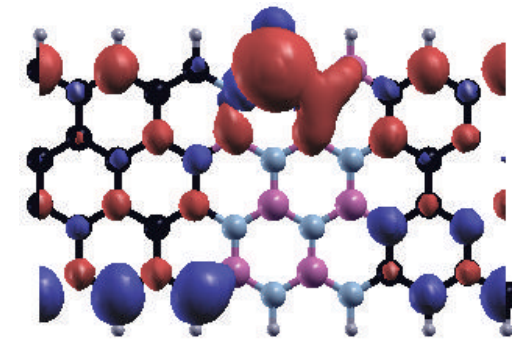

(a)

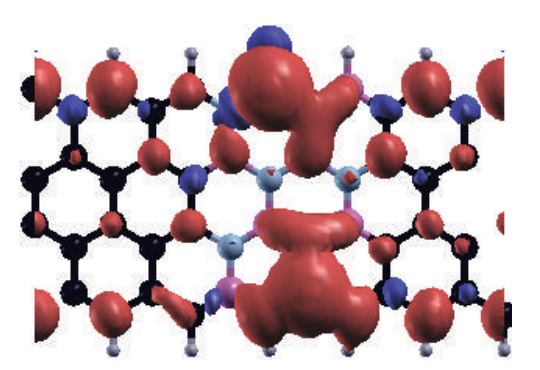

(b)

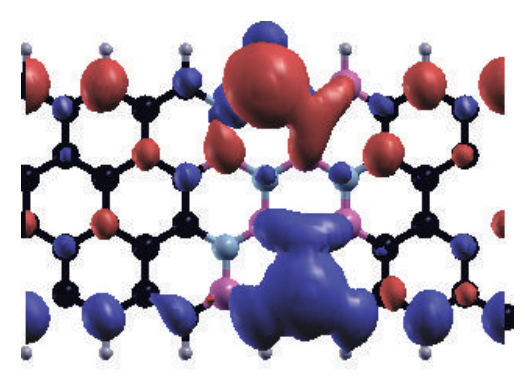

(c)

FIgure 2: Spin densities for the structures with one Mn impurity (a) and two Mn impurities in ferromagnetic (b) and antiferromagnetic (c) configurations at zero bias.

the spin current only. The on-off transition observed in the spin current is analogous to the one found in a conventional field-effect transistor, where the switching behavior of the charge current is one of the most important performance parameters. By contrast, the sample with two magnetic impurities shows a polarization above $10 \%$ even at lowest bias considered for both spin configurations. Relatively high spin injection rates (above 50\%) are obtained at rather small bias, which indicates a suitable behavior for a spin-filter device. Up to the bias $U=0.6 \mathrm{~V}$ for both ferromagnetic and antiferromagnetic configurations there is a similar behavior, which indicates the reliability of the spin-filter characteristics under the change (flip) of the magnetic moments present, which may occur in a realistic device subject to thermal fluctuations. For even larger biases $(U>0.6 \mathrm{~V})$, the spin current polarization begins to drop for the FM configuration, while for AFM configuration the values for the polarization become as large as $p \approx 0.85$ for $U=1 \mathrm{~V}$. 

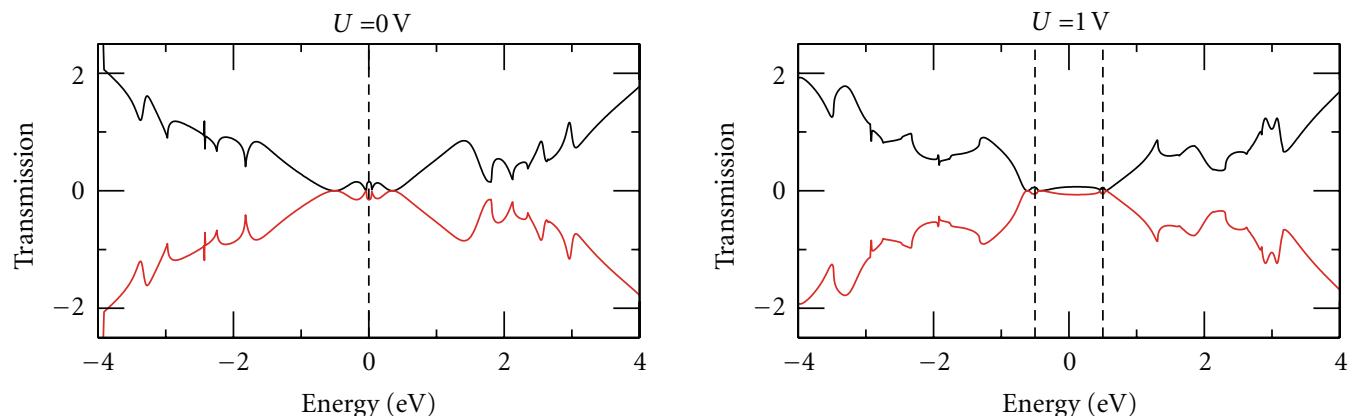

(a)
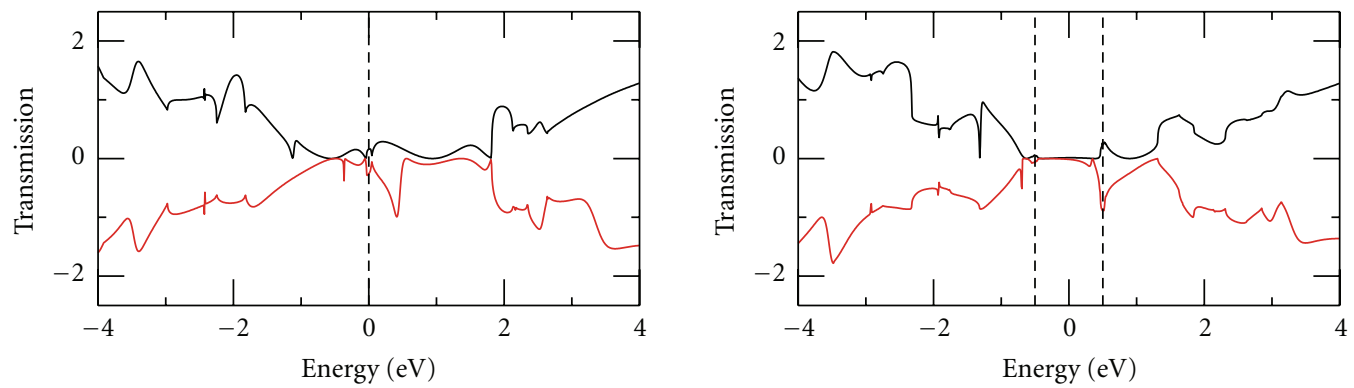

(b)
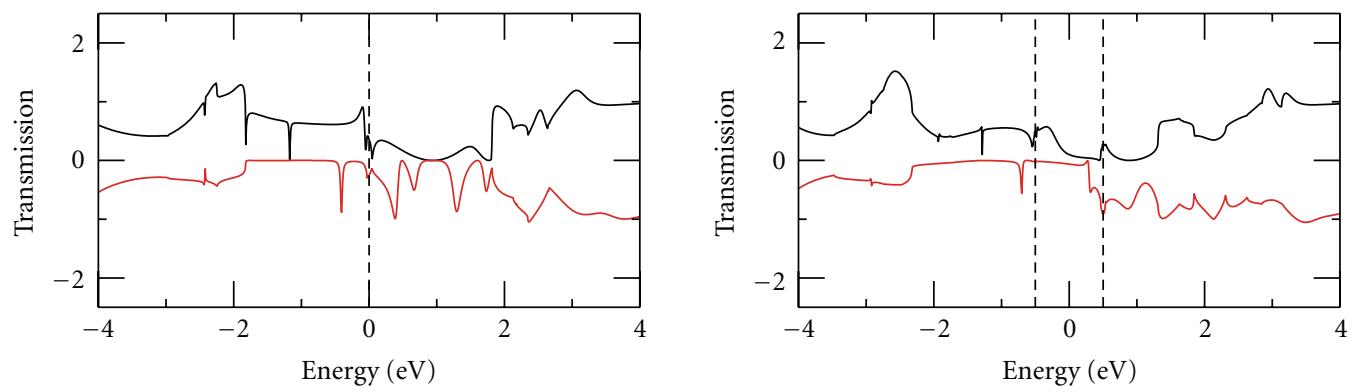

(c)
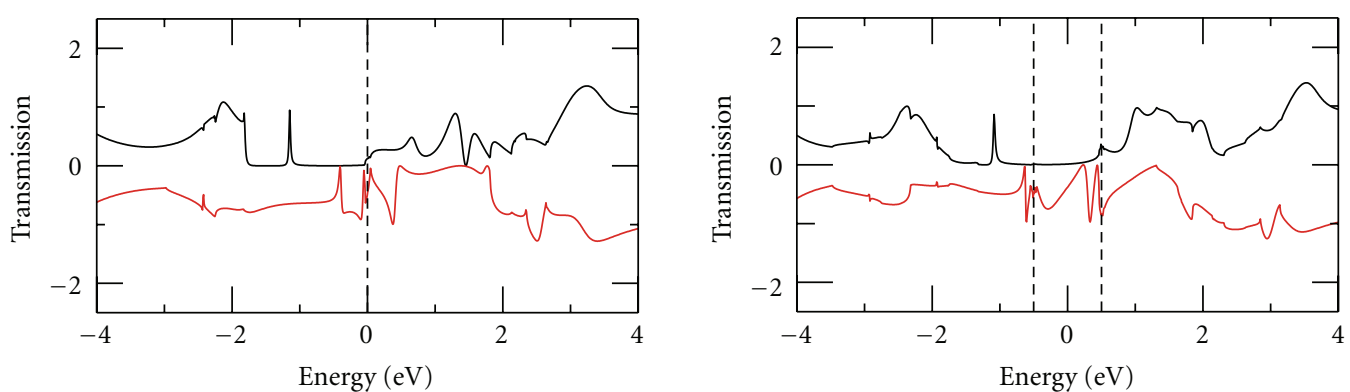

(d)

Figure 3: Transmission versus energy $T(E)$ for pristine zBNNR (a), one Mn impurity (b), two Mn impurities in ferromagnetic configuration (c), and Mn impurities in antiferromagnetic configuration (d), for zero bias and for $U=1 \mathrm{~V}(V=1 \mathrm{eV})$. Spin-up (black) and spin-down (red) components are indicated. The vertical dashed lines mark the energy window from (1).

\section{Conclusions}

The spin transport properties were investigated in boron nitride nanoribbons with one or two substitutional $\mathrm{Mn}$ impurities, connected to graphene electrodes. The structure with one magnetic impurity suggests potential applications for a spin current switch: at lower biases, the spin current is negligible (off state), followed by clear onset in the spin polarization (on state). The sample containing two Mn atoms proves to be suitable for spin-filter applications. On one hand, up to intermediate biases, there is a reliable behavior of the spin current polarization, independent of the configurations of the magnetic moments (FM or AFM). On the other hand, for the AFM configuration, at even 


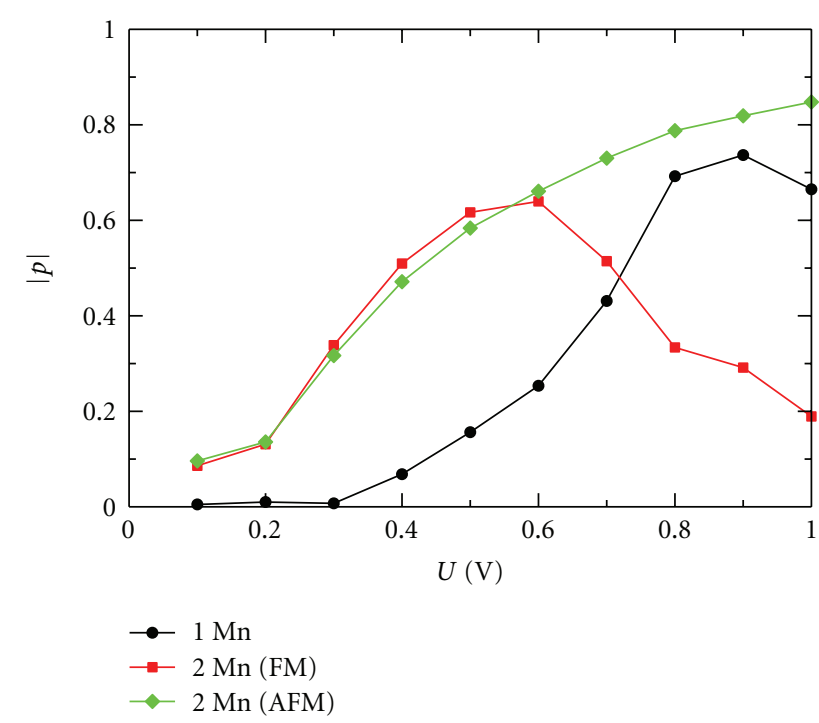

FIGURE 4: Spin current polarization for magnetic systems with one and two Mn impurities. For the system with two Mn impurities results for both ferromagnetic (FM) and antiferromagnetic (AFM) configurations are presented.

larger biases the polarization is enhanced significantly. The building blocks (i.e., single layer BN, graphene, or mixtures) of the structures presented here are already experimentally produced. Such ultrathin devices based on low-dimensional diluted magnetic semiconductors may offer the possibility to extend their operation to room temperature, which makes them strong candidates for the future spintronic technology.

\section{Acknowledgment}

This work was supported by the Romanian National Authority for Scientic Research, CNCS-UEFISCDI, Project no. PNII-RU-PD-2011-3-0044.

\section{References}

[1] D. Ma, Z. Lu, W. Ju, and Y. Tang, "First-principles studies of $\mathrm{BN}$ sheets with absorbed transition metal single atoms or dimers: stabilities, electronic structures, and magnetic properties," Journal of Physics Condensed Matter, vol. 24, no. 14, Article ID 145501, 2012.

[2] T. L. Mitran, A. Nicolaev, G. A. Nemnes, L. Ion, and S. Antohe, "Magnetic behavior and clustering effects in Mn-doped boron nitride sheets," Journal of Physics Condensed Matter, vol. 24, no. 32, Article ID 326003, 2012.

[3] K. S. Novoselov, D. Jiang, F. Schedin et al., "Two-dimensional atomic crystals," Proceedings of the National Academy of Sciences of the United States of America, vol. 102, no. 30, pp. 10451-10453, 2005.

[4] D. Paciĺ, J. C. Meyer, C. Girit, and A. Zettl, "The twodimensional phase of boron nitride: few-atomic-layer sheets and suspended membranes," Applied Physics Letters, vol. 92, no. 13, Article ID 133107, 2008.

[5] C. Lee, Q. Li, W. Kalb et al., "Frictional characteristics of atomically thin sheets," Science, vol. 328, no. 5974, pp. 76-80, 2010.
[6] N. Alem, R. Erni, C. Kisielowski, M. D. Rossell, W. Gannett, and A. Zettl, "Atomically thin hexagonal boron nitride probed by ultrahigh-resolution transmission electron microscopy," Physical Review B, vol. 80, no. 15, Article ID 155425, 2009.

[7] C. Jin, F. Lin, K. Suenaga, and S. Iijima, "Fabrication of a freestanding boron nitride single layer and Its defect assignments," Physical Review Letters, vol. 102, no. 19, Article ID 195505, 2009.

[8] C. Zhi, Y. Bando, C. Tang, H. Kuwahara, and D. Golberg, "Large-scale fabrication of boron nitride nanosheets and their utilization in polymeric composites with improved thermal and mechanical properties," Advanced Materials, vol. 21, no. 28, pp. 2889-2893, 2009.

[9] A. Nag, K. Raidongia, K. P. S. S. Hembram, R. Datta, U. V. Waghmare, and C. N. R. Rao, "Graphene analogues of BN: novel synthesis and properties," ACS Nano, vol. 4, no. 3, pp. 1539-1544, 2010.

[10] L. Song, L. Ci, H. Lu et al., "Large scale growth and characterization of atomic hexagonal boron nitride layers," Nano Letters, vol. 10, no. 8, pp. 3209-3215, 2010.

[11] L. Ci, L. Song, C. Jin et al., "Atomic layers of hybridized boron nitride and graphene domains," Nature Materials, vol. 9, no. 5, pp. 430-435, 2010.

[12] E. A. Basheer, P. Parida, and S. K. Pati, "Electronic and magnetic properties of BNC nanoribbons: a detailed computational study," New Journal of Physics, vol. 13, Article ID 053008, 2011.

[13] V. Barone, O. Hod, and G. E. Scuseria, "Electronic structure and stability of semiconducting graphene nanoribbons," Nano Letters, vol. 6, no. 12, pp. 2748-2754, 2006.

[14] J. M. Soler, E. Artacho, J. D. Gale et al., "The SIESTA method for $\mathrm{ab}$ initio order-N materials simulation," Journal of Physics Condensed Matter, vol. 14, no. 11, pp. 2745-2779, 2002.

[15] M. Brandbyge, J. L. Mozos, P. Ordejón, J. Taylor, and K. Stokbro, "Density-functional method for nonequilibrium electron transport," Physical Review B, vol. 65, no. 16, Article ID 165401, 2002. 

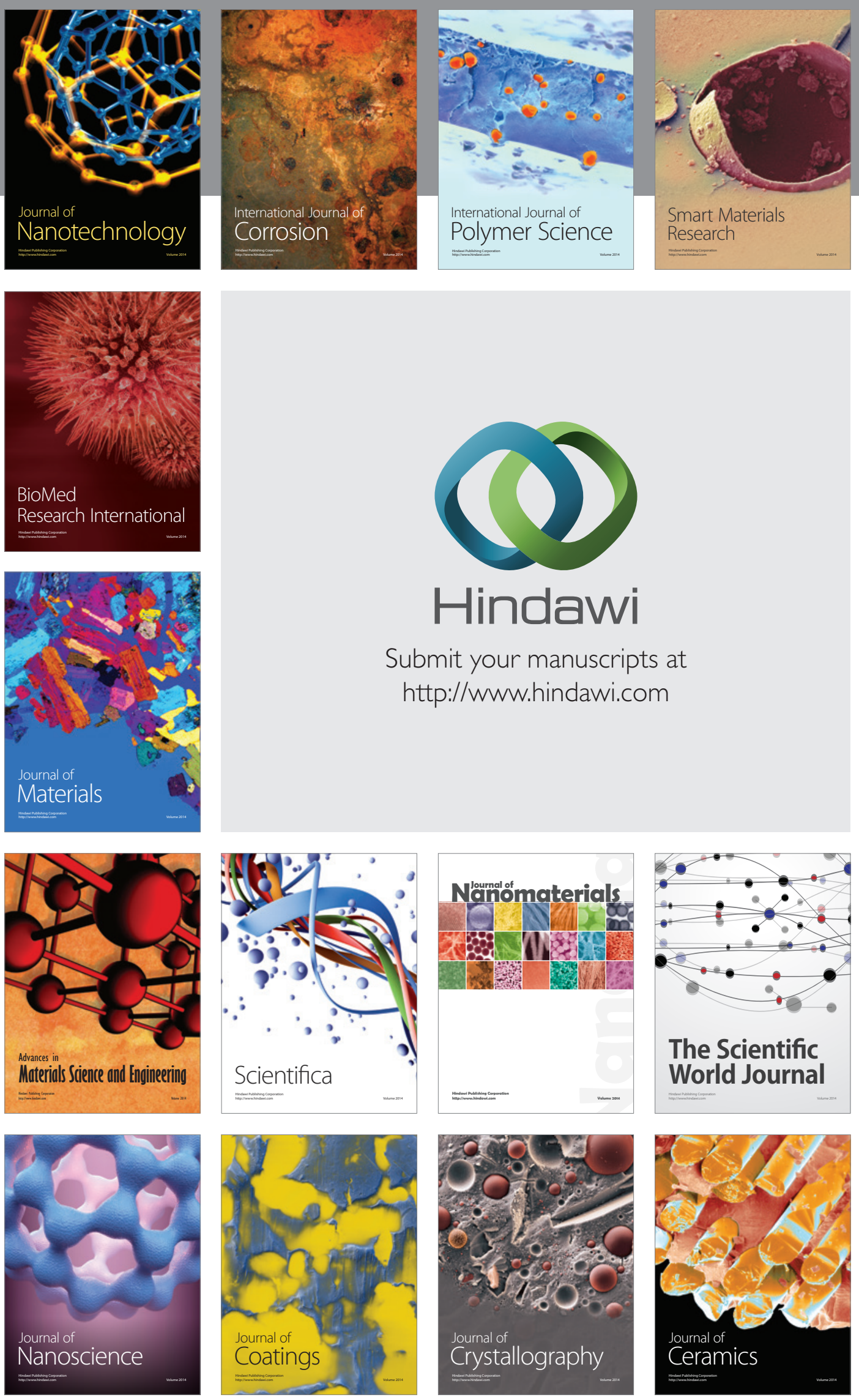

The Scientific World Journal

Submit your manuscripts at

http://www.hindawi.com

\section{World Journal}

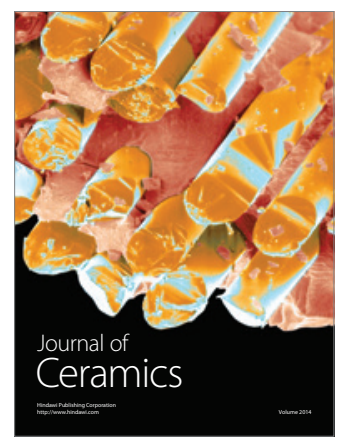

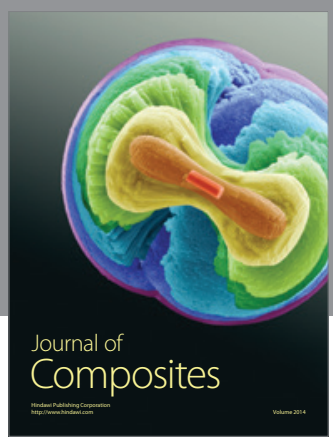
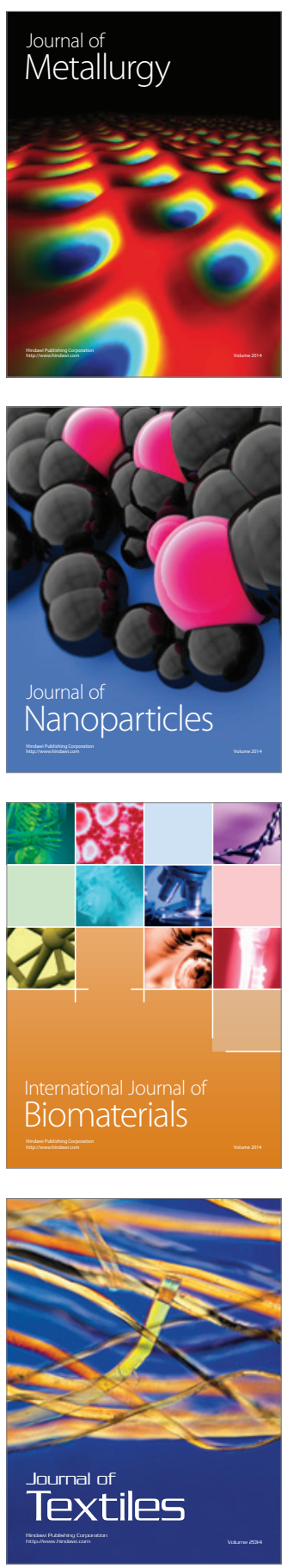